\title{
Caracterización morfológica del chicozapote (Manilkara zapota) del centro de Veracruz y Morelos, México: base para su mejoramiento
}

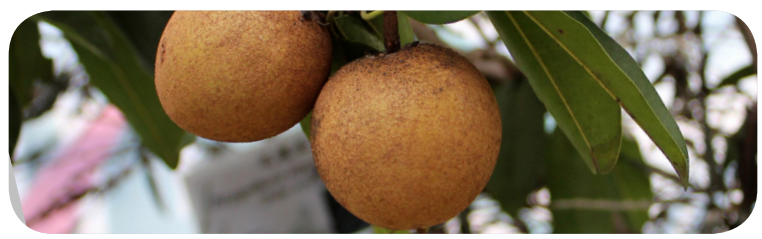

\author{
Víctor Heber Aguilar Rincón ${ }^{1}$ \\ Tarsicio Corona Torres ${ }^{2}$ \\ Pedro Juárez González $z^{3}$ \\ Juan A. Villanueva Jiménez $z^{4}$
}

Recepción: 28/05/2018 Aceptación: 11/08/2018

\section{Resumen}

En el estado de Veracruz y Morelos, México, el Manilkara zapota se utiliza para la producción del fruto. Además, existen árboles que evidencian haber sido explotados para la extracción del látex. El objetivo de la investigación fue determinar qué variables morfológicas son las más adecuadas para caracterizar los materiales cultivados y criollos de chicozapote presentes en la región centro del Estado de Veracruz y del Estado de Morelos, con la finalidad de revisar su potencial como material fuente para el desarrollo de nuevas variedades.

Se evaluaron varios rasgos morfológicos de la hoja, flor, fruto y tallo mediante técnicas multivariadas (análisis de componentes principales, análisis canónico discrimínate y análisis clúster). Las variables que resultaron más sobresalientes para caracterizar al material de chicozapote evaluado fueron el ancho de hoja, flores por racimo, ancho de fruto, longitud del pedúnculo del fruto y peso del fruto. Varios árboles criollos presentaron características relevantes con calidad excepcional al fruto, tales como forma ovalada o semiovalada; color café claro, poco rugoso, con pulpa café rojiza sin grumos, tamaño mediano a grande y un número escaso de semillas, los cuales deben rescatarse para formar variedades mejoradas autóctonas.

Palabras clave: Análisis multivariado, caracteres botánicos, diversidad biológica, rescate de recursos naturales.

\begin{abstract}
In the state of Veracruz and Morelos, Mexico, the Manilkara zapota is used for fruit production. In addition, there are trees that show that they have been exploited for the extraction of latex. The objective of the research was to determine which morphological variables are the most appropriate to characterize the cultivated and creole materials of chicozapote present in the central region of the State of Veracruz and the State of Morelos, in order to review their potential as source material for the development of new varieties.
\end{abstract}

Several morphological features of the leaf, flower, fruit and stem were evaluated by multivariate techniques (analysis of principal components, discriminant canonical analysis and cluster analysis). The variables that were most outstanding to characterize the evaluated chicozapote material were leaf width, flowers per bunch, width of fruit, length of peduncle of the fruit and weight of the fruit. Several Creole trees presented outstanding characteristics with exceptional quality to the fruit, such as oval or semioval shape; light brown color, slightly rough, with reddish brown pulp without lumps, medium to large size and a limited number of seeds, which must be rescued to form autochthonous improved varieties.

Key words: Multivariate analysis, botanical characters, biological diversity, rescue of natural resources.

1. Doctor en Ciencias, Profesor Investigador, Colegio de Postgraduados, Campus Montecillo; Texcoco, Edo. de México, México; email: aheber@colpos.mx

2. Doctor en Ciencias, Profesor Investigador, Colegio de Postgraduados, Campus Montecillo; Texcoco, Edo. de México, México; email: tcoronat@colpos.mx

3. Maestro en Ciencias, Docente, Centro de Bachillerato Tecnológico Agropecuario - No. 277, Villa Emiliano Zapata, Veracruz, México; email: peju485@hotmail.com

4. Doctor en Ciencias, Profesor Investigador, Colegio de Postgraduados, Campus Veracruz - Manlio F. Altamirano, Veracruz, México; email: javj@colpos.mx // Responsable de la publicación. 


\section{Introducción}

La variedad de climas en combinación con la heterogeneidad física y geográfica de México han desarrollado buena parte de la biodiversidad donde el hombre ha incidido al utilizar diferentes especies y domesticarlas. Esta reserva de diversidad de recursos naturales permite amortiguar los cada vez más frecuentes cambios ambientales, y aquellos impulsados por la presión sobre los recursos hecha por el hombre, tales como la deforestación, urbanización, erosión, entre otros. Además, la pérdida del material natural amenaza la seguridad alimentaria de los pueblos (Ramírez et al., 2000).

Los recursos genéticos de México son de especial interés, como es el caso de especies que eran ampliamente utilizadas en el México prehispánico (Herron, 1999); y que después se volvieron económicamente importantes, como lo es el chicozapote (Manilkara zapota (L.) P. Royen (1953)). Este árbol originario de Mesoamérica (Hernández y León, 1992) presenta tolerancia a las condiciones de sequía, es resistente a los vientos fuertes y se adapta bien a los suelos calizos (García et al., 1983); lo que le confiere ventajas sobre otros frutales tropicales perennes (Rzedowski, 1988). Esta especie se ha aprovechado sistemáticamente desde 1915 para la extracción de látex y producción de chicle; su madera es de excepcional dureza y sus frutos son muy apreciados por su sabor (Ramírez et al., 2000).
En la región centro del Estado de Veracruz, esta especie se utiliza para la producción del fruto en regiones tales como ApazapanAgua Caliente, Emiliano Zapata-Plan del Río y Cuajilote-San José Chipila donde el fruto criollo se comercializa en los mercados regionales, y representa una fuente de ingreso para los productores. Otros usos de esta especie son la reforestación de terrenos desmontados y abandonados, en donde previene la erosión hídrica del suelo, conserva el recurso agua en las cuencas y preserva la fauna silvestre (Toledo, 1982). Es posible que entre estos materiales criollos existan frutos con características de calidad potencial para mercados especializados. Sin embargo, la introducción de la variedad Betawi de Indonesia en el siglo pasado, con un fruto grande y buen sabor, desplazó al fruto criollo en el ámbito comercial (Báez, 1979).

Por otro lado, para hacer una buena utilización per se o en un programa de mejoramiento de un recurso genético, es indispensable tener un conocimiento lo más preciso de él, ya sea a través de sus características morfológicas, químicas y moleculares. De estas metodologías, la morfológica es el primer paso para introducirse en el conocimiento de su diversidad, para lo cual es necesario considerar la mayoría de sus características de planta, hoja, flor y fruto; y aplicar metodologías multivariadas que nos permitan caracterizar, diferenciar y agrupar individuos o poblaciones 
con mayor similitud. Sobre la base anterior, la presente investigación tuvo como objetivo caracterizar morfológicamente los árboles de chicozapote criollo del centro del Estado de Veracruz y del Estado de Morelos en México.

\section{Materiales y Métodos}

Para la colecta, se ubicaron las áreas de Apazapan-Agua Caliente, Emiliano Zapata-Plan del Río y Cuajilote-San José Chipila del Estado de Veracruz, México. Debido a que existen huertas comerciales con la variedad mejorada Betawi, importada del Sureste Asiático, éstas fueron incluidas en el estudio para contrastar la información obtenida de los materiales criollos. También se obtuvo información de árboles presentes en la localidad Tlalquitenango, Morelos, México.

Se buscó incluir en el estudio los árboles con el mayor perímetro de sus tallos, con lo que se asumió que eran los árboles de mayor edad. No se registró el diámetro de los árboles muestreados en Morelos. Cada sitio de colecta fue inspeccionado hasta cuatro veces, en virtud de la gran variabilidad de épocas de floración y fructificación. Se colectaron ramas de los cuatro puntos cardinales de cada árbol, los cuales contenían hojas, flores y frutos. Las variables registradas fueron las siguientes: altura medida con un clisímetro y perímetro del árbol con cinta métrica (Tablas
1 y 2); coordenadas geográficas y altura sobre el nivel del mar medidos con un GPS X12 ${ }^{\mathrm{MR}}$.

Las siguientes variables de hojas, flores, frutos y semillas conformaron la base de datos para el análisis multivariado: número de hojas por racimo, longitud del pedicelo, largo y ancho de hoja; forma, textura, color y posición las hojas. Número de flores por racimo, sépalos y pétalos, color de pétalos, diámetro del botón floral y de la flor, longitud de la flor y del pedicelo. Longitud y ancho del fruto, longitud de su pedicelo, peso del fruto y grados brix. Forma, color y textura de fruto y pulpa. Número de semillas por fruto, largo, ancho, color, textura y peso de la semilla. Los diámetros se midieron con vernier (Scala, México ${ }^{\mathrm{MR}}$ ), longitudes con regla graduada y los grados brix con refractómetro manual.

Se realizó un análisis de componentes principales $\left(\mathrm{ACP}^{5}\right)$ con una matriz de correlación, por existir diferentes escalas en los caracteres. La selección de las variables de mayor importancia en la variación de las poblaciones se llevó a cabo con base en los valores máximos de los vectores propios y por el coeficiente de correlación $(\geq 0.7)$, para el primer y tercer componente principal obtenido; para el segundo componente se empleó el coeficiente de correlación de $(\geq 0.6)$. El coeficiente de correlación (CC) 5 se calculó con la fórmula CC

5. Dentro del documento, los autores también se referirán a este término mediante su acrónimo. 
$=(\lambda) 2 \cdot \mathrm{CP}$, donde $\lambda$ es el valor propio $\mathrm{y} \mathrm{CP}$ es el valor de los vectores propios de cada componente principal.

La representación gráfica de la dispersión de las 45 colectas se obtuvo para los dos primeros componentes principales. Para caracterizar las colectas de chicozapote, se probó la hipótesis que las colectas se separan en grupos diferentes utilizando los caracteres seleccionados en el ACP, éstos fueron sometidos a un análisis canónico discriminante (ACD). Con los valores de la matriz simple estandarizada, fueron identificadas las variables de mayor importancia en la formación de los grupos. Para determinar diferencias estadísticas entre los grupos, éstos fueron evaluados a través de la distancia de "Mahalanobis". La representación gráfica de los grupos se hizo con las funciones discriminantes (1 y 2) que mejor explicaron la varianza observada.
Las 45 colectas de chicozapote fueron reagrupadas mediante el método de pares de grupo con más peso, mediante la media aritmética (UPGMA) del paquete estadístico NTSYSpc, Numerical Taxonomy System versión $2.2^{6}$. Previo al agrupamiento, se estandarizó la matriz de datos y se calculó la matriz de similitud mediante el coeficiente de distancia.

\section{Resultados y Discusión}

En la Tabla 1 se observa una relación directa entre el perímetro del árbol y la altura del mismo en las áreas de colecta de chicozapote. En Apazapan se presentan los mayores tamaños, seguida de los de Cuajilote. La variedad Betawi fue representada por árboles de 40 años de edad con perímetro reducido y altura promovida por condiciones de riego y manejo que les permite desarrollarse mejor. La Tabla 2 presenta los datos de georreferenciación de colecta de los materiales, en altitudes que van de los 150 a 550m. (Ver tabla 1 ).

Tabla 1. Perímetro y altura de árboles de chicozapote criollos y cultivados, muestreados en el Centro de Veracruz, México

\begin{tabular}{|c|c|c|c|c|c|c|}
\hline & Perímetro & Máximo & Mínimo & $\begin{array}{c}\text { Altura } \\
\text { del árbol }\end{array}$ & Máximo & Mínimo \\
\hline Localidad (cultivar) & \multicolumn{7}{|l}{} \\
\hline Agazapan (criollo) & 3.45 & 5.07 & 1.93 & 27.25 & 55.06 & 9.75 \\
\hline Cuajilote (criollo) & 2.86 & 4.77 & 2.17 & 18.00 & 27.77 & 9.37 \\
\hline E. Zapata (criollo) & 2.08 & 3.47 & 0.67 & 13.71 & 18.65 & 8.66 \\
\hline E. Zapata (var. Betawi) & 0.89 & 0.93 & 0.85 & 13.96 & 14.34 & 13.58 \\
\hline
\end{tabular}

6. Disponible en http://www.exetersoftware.com/cat/ntsyspc/ntsyspc.html 
Tabla 2. Localización en coordenadas geográficas y metros sobre el nivel del mar y clave de los árboles muestreados

\begin{tabular}{|c|c|c|c|c|c|c|c|}
\hline Clave & Latitud Norte & $\begin{array}{c}\text { Longitud } \\
\text { Oeste }\end{array}$ & msnm & Clave & Latitud Norte & $\begin{array}{c}\text { Longitud } \\
\text { Oeste }\end{array}$ & msnm \\
\hline \multicolumn{8}{|c|}{ Localidad Apazapan } \\
\hline A1 & $19^{\circ} 19^{\prime} 57.06^{\prime \prime}$ & $96^{\circ} 43^{\prime} 48.98^{\prime \prime}$ & 361 & A9 & $19^{\circ} 20^{\prime} 10.62^{\prime \prime}$ & $96^{\circ} 43^{\prime} 15.72^{\prime \prime}$ & 372 \\
\hline A2 & $19^{\circ} 19^{\prime} 58.80^{\prime \prime}$ & $96^{\circ} 43^{\prime} 35.46^{\prime \prime}$ & 352 & A10 & $19^{\circ} 20^{\prime} 08.46^{\prime \prime}$ & $96^{\circ} 43^{\prime} 17.10^{\prime \prime}$ & 356 \\
\hline A3 & $19^{\circ} 19^{\prime} 58.56^{\prime \prime}$ & $96^{\circ} 43^{\prime} 37.56^{\prime \prime}$ & 349 & A11 & $19^{\circ} 20^{\prime} 01.38$ & $96^{\circ} 43^{\prime} 17.22^{\prime \prime}$ & 346 \\
\hline A4 & $19^{\circ} 19^{\prime} 58.26^{\prime \prime}$ & $96^{\circ} 43^{\prime} 37.62^{\prime \prime}$ & 333 & A12 & $19^{\circ} 19^{\prime} 54.48^{\prime \prime}$ & $96^{\circ} 43^{\prime} 25.20^{\prime \prime}$ & 338 \\
\hline A5 & $19^{\circ} 19^{\prime} 47.70^{\prime \prime}$ & $96^{\circ} 43^{\prime} 36.00^{\prime \prime}$ & 323 & A13 & $19^{\circ} 19^{\prime} 54.84^{\prime \prime}$ & $96^{\circ} 43^{\prime} 25.74^{\prime \prime}$ & 336 \\
\hline A6 & $19^{\circ} 19^{\prime} 48.90^{\prime \prime}$ & $96^{\circ} 43^{\prime} 35.70^{\prime \prime}$ & 328 & A14 & $19^{\circ} 19^{\prime} 54.54^{\prime \prime}$ & $96^{\circ} 43^{\prime} 26.82^{\prime \prime}$ & 340 \\
\hline A7 & $19^{\circ} 19^{\prime} 44.28^{\prime \prime}$ & $96^{\circ} 43^{\prime} 32.88^{\prime \prime}$ & 327 & A15 & $19^{\circ} 19^{\prime} 54.48^{\prime \prime}$ & $96^{\circ} 43^{\prime} 28.26^{\prime \prime}$ & 343 \\
\hline A8 & $19^{\circ} 20^{\prime} 11.58^{\prime \prime}$ & $96^{\circ} 43^{\prime} 12.60^{\prime \prime}$ & 383 & A16 & $19^{\circ} 19^{\prime} 51.18^{\prime \prime}$ & $96^{\circ} 43^{\prime} 40.74^{\prime \prime}$ & 317 \\
\hline \multicolumn{8}{|c|}{ Localidad Agua Caliente } \\
\hline G1 & $19^{\circ} 19^{\prime} 18.66^{\prime \prime}$ & $96^{\circ} 38^{\prime} 15.78^{\prime \prime}$ & 228 & G3 & $19^{\circ} 19^{\prime} 18.66^{\prime \prime}$ & $96^{\circ} 38^{\prime} 15.78^{\prime \prime}$ & 225 \\
\hline G2 & $19^{\circ} 19^{\prime} 18.66^{\prime \prime}$ & $96^{\circ} 38^{\prime} 15.78^{\prime \prime}$ & 227 & & & & \\
\hline \multicolumn{8}{|c|}{ Localidad Emiliano Zapata } \\
\hline Z1 & $19^{\circ} 24^{\prime} 38.88^{\prime \prime}$ & $96^{\circ} 40^{\prime} 36.30^{\prime \prime}$ & 411 & $\mathrm{Z7}$ & $19^{\circ} 23^{\prime} 52.26^{\prime \prime}$ & $96^{\circ} 38^{\prime} 49.92^{\prime \prime}$ & 296 \\
\hline $\mathbf{Z 2}$ & $19^{\circ} 24^{\prime} 40.92^{\prime \prime}$ & $96^{\circ} 40^{\prime} 36.24^{\prime \prime}$ & 417 & Z8 & $19^{\circ} 23^{\prime} 52.26^{\prime \prime}$ & $96^{\circ} 38^{\prime} 49.92^{\prime \prime}$ & 296 \\
\hline Z3 & $19^{\circ} 24^{\prime} 40.80^{\prime \prime}$ & $96^{\circ} 40^{\prime} 35.58^{\prime \prime}$ & 420 & Z9 & $19^{\circ} 23^{\prime} 51.84^{\prime \prime}$ & $96^{\circ} 38^{\prime} 57.36^{\prime \prime}$ & 296 \\
\hline $\mathrm{Z4}$ & $19^{\circ} 24^{\prime} 04.80^{\prime \prime}$ & $96^{\circ} 38^{\prime} 58.44^{\prime \prime}$ & 288 & Z10 & $19^{\circ} 23^{\prime} 52.44^{\prime \prime}$ & $96^{\circ} 38^{\prime} 57.18^{\prime \prime}$ & 296 \\
\hline $\mathrm{Z5}$ & $19^{\circ} 24^{\prime} 04.86^{\prime \prime}$ & $96^{\circ} 38^{\prime} 58.08^{\prime \prime}$ & 290 & Z11 & $19^{\circ} 22^{\prime} 09.78^{\prime \prime}$ & $96^{\circ} 39^{\prime} 27.54^{\prime \prime}$ & 420 \\
\hline Z6 & $19^{\circ} 24^{\prime} 05.34^{\prime \prime}$ & $96^{\circ} 38^{\prime} 59.94^{\prime \prime}$ & 290 & & & & \\
\hline \multicolumn{8}{|c|}{ Localidad Cuajilote } \\
\hline $\mathrm{Cl}$ & $19^{\circ} 18^{\prime} 27.78^{\prime \prime}$ & $96^{\circ} 34^{\prime} 22.08^{\prime \prime}$ & 150 & $\mathrm{C5}$ & $19^{\circ} 18^{\prime} 43.38^{\prime \prime}$ & $96^{\circ} 34^{\prime} 38.40^{\prime \prime}$ & 179 \\
\hline $\mathrm{C} 2$ & $19^{\circ} 18^{\prime} 44.76^{\prime \prime}$ & $96^{\circ} 34^{\prime} 42.36^{\prime \prime}$ & 196 & C6 & $19^{\circ} 17^{\prime} 54.48^{\prime \prime}$ & $96^{\circ} 34^{\prime} 47.16^{\prime \prime}$ & 174 \\
\hline $\mathrm{C} 3$ & $19^{\circ} 18^{\prime} 43.62^{\prime \prime}$ & $96^{\circ} 34^{\prime} 39.78^{\prime \prime}$ & 169 & $\mathrm{C7}$ & $19^{\circ} 17^{\prime} 52.44^{\prime \prime}$ & $96^{\circ} 35^{\prime} 03.06^{\prime \prime}$ & 153 \\
\hline $\mathrm{C4}$ & $19^{\circ} 18^{\prime} 44.94^{\prime \prime}$ & $96^{\circ} 34^{\prime} 46.68^{\prime \prime}$ & 180 & $\mathrm{C} 8$ & $19^{\circ} 17^{\prime} 54.60^{\prime \prime}$ & $96^{\circ} 35^{\prime} 09.42^{\prime \prime}$ & 160 \\
\hline \multicolumn{4}{|c|}{ Localidad Tlalquitenango, Morelos, México } & \multicolumn{4}{|c|}{ Variedad Betawi, Localidad Emiliano Zapata } \\
\hline $\begin{array}{l}\text { M1, } \\
\text { M2 }\end{array}$ & \multirow{3}{*}{\multicolumn{2}{|c|}{$\begin{array}{c}\text { Muestras: LN } 18^{\circ} 54^{\prime} 19.20^{\prime \prime} \\
\text { LO } 99^{\circ} 05^{\prime} 19.8^{\prime \prime}\end{array}$}} & \multirow{2}{*}{$\begin{array}{c}\text { De } \\
450 \mathrm{a} \\
550\end{array}$} & B1 & $19^{\circ} 23^{\prime} 52.74^{\prime \prime}$ & $96^{\circ} 38^{\prime} 57.40^{\prime \prime}$ & 298 \\
\hline $\begin{array}{l}\text { M3, } \\
\text { M4 }\end{array}$ & & & & B2 & $19^{\circ} 23^{\prime} 51.60^{\prime \prime}$ & $96^{\circ} 38^{\prime} 49.85^{\prime \prime}$ & 305 \\
\hline M5 & & & & & & & \\
\hline
\end{tabular}


Se coincide con diferentes autores en que la variabilidad de la altura del árbol de chicozapote criollo (13.75 a $27.25 \mathrm{~m}$, Tabla 1$)$ depende del área natural donde se desarrollen los árboles. León (1968) indica que el árbol de chicozapote criollo mide de 5 a $15 \mathrm{~m}$ de alto; sin embargo, Roecklein y Leung (1987) reportan alturas de hasta $38 \mathrm{~m}$. Castner et al. (1998), indican que es un árbol siempre verde de un tamaño medio (15 a 30m); Ortega et al. (2000) reportan una altura de 40m y Batis et al., (1999) una de hasta 45m.

El diámetro de los árboles criollos en el área de estudio fue de 0.66 a $1.10 \mathrm{~m}$, aunque dos árboles midieron de 1.59 y $1.61 \mathrm{~m}$. Batis et al., (1999) indican un diámetro máximo a la altura de pecho de hasta $1.25 \mathrm{~m}$, lo cual es una medida intermedia de las obtenidas en el estudio.

En las Figuras 1, 2, 3 y 4 se observa mayor tamaño en los rasgos botánicos en árboles de Emiliano Zapata-Plan del Río y Apazapan-Agua Caliente, en comparación con la región de Cuajilote, donde sobresalen las variables de hojas por racimo y peso de fruto. (Ver figura 1).

Sin embargo, los rasgos botánicos importantes para la comercialización, como peso de fruto, color de pulpa y grados de sacarosa, permiten caracterizar a la región ApazapanAgua Caliente por una mejor calidad de fruto en los materiales criollos de chicozapote; en la región de Cuajilote-San José Chipila, los rasgos botánicos con mayores medidas no son relevantes, aunque se pudieron encontrar algunos árboles criollos interesantes, como un árbol prácticamente sin semilla (C3), otro con una alta calidad de fruto (C4), o de un gran peso de fruto (C7), (ver figura 2).

Las variables categóricas de forma, color, textura del fruto y de la pulpa, y color de la semilla, presentaron suficientes diferencias, dentro y entre las regiones de colecta, que permitieron caracterizar al fenotipo. En contraste, se observó que las variables categóricas de forma, textura, color y posición de la hoja, color del pétalo y textura de la semilla no son relevantes para la caracterización del mismo. (Ver figuras 3 y 4 ).

Se concuerda con Castner et al. (1998) que incluso en tiempo de estiaje, M. zapota presenta frutos disponibles para recolectar, lo que aporta un papel adicional de este frutal en la alimentación no solo del hombre, sino de distintas especies de mamíferos y aves (Toledo, 1982).

Los valores del peso de la matriz de correlación en el análisis de componentes principales (ACP) dieron como resultado tres componentes, con una explicación acumulada de 0.626 (Tabla 3), lo cual es considerado como aceptable para definir los componentes de interés en la caracterización de materiales biológicos. 


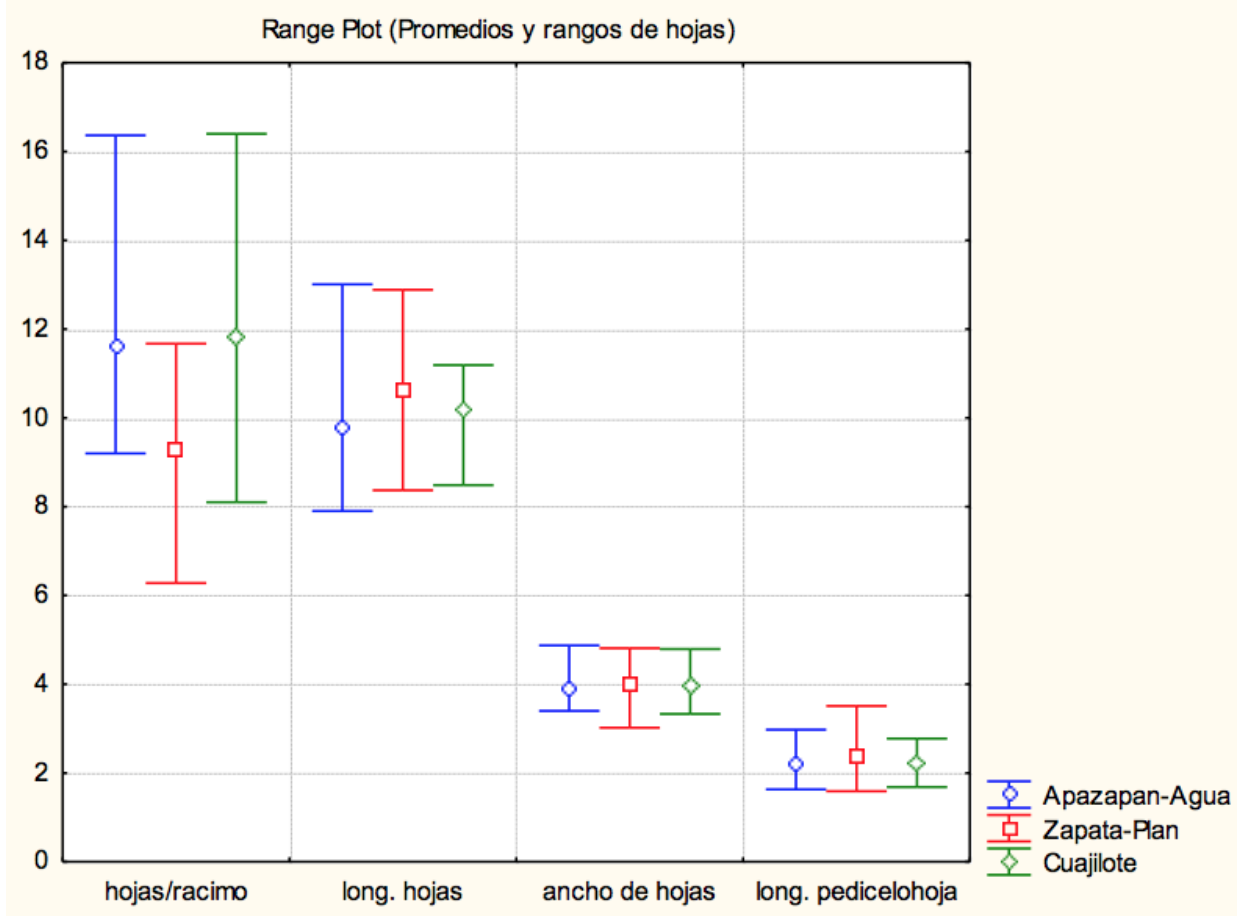

Figura 1. Promedios y rangos por región de colecta, de las variables numéricas de hojas de chicozapote.

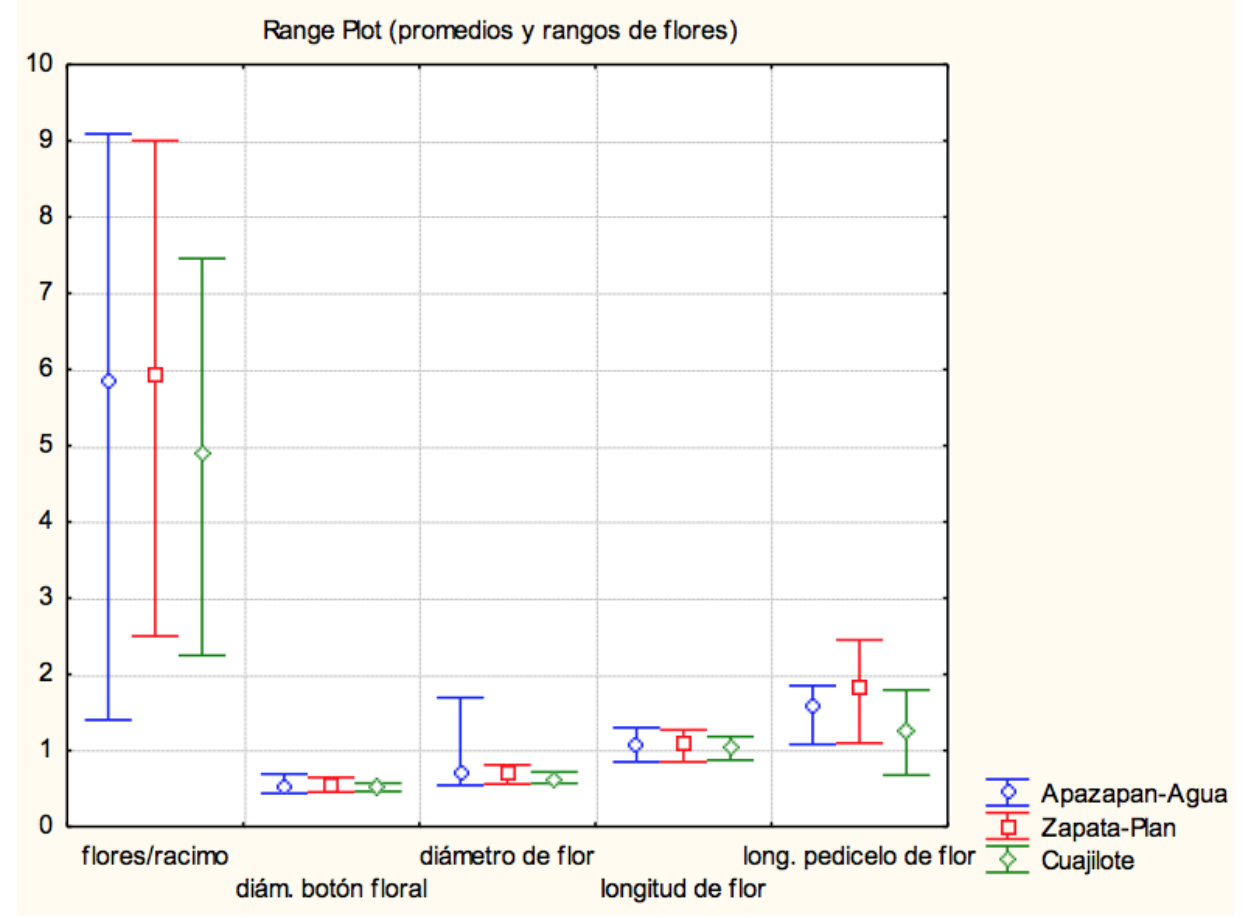

Figura 2. Promedios y rangos por región de colecta, de las variables numéricas de flores. 


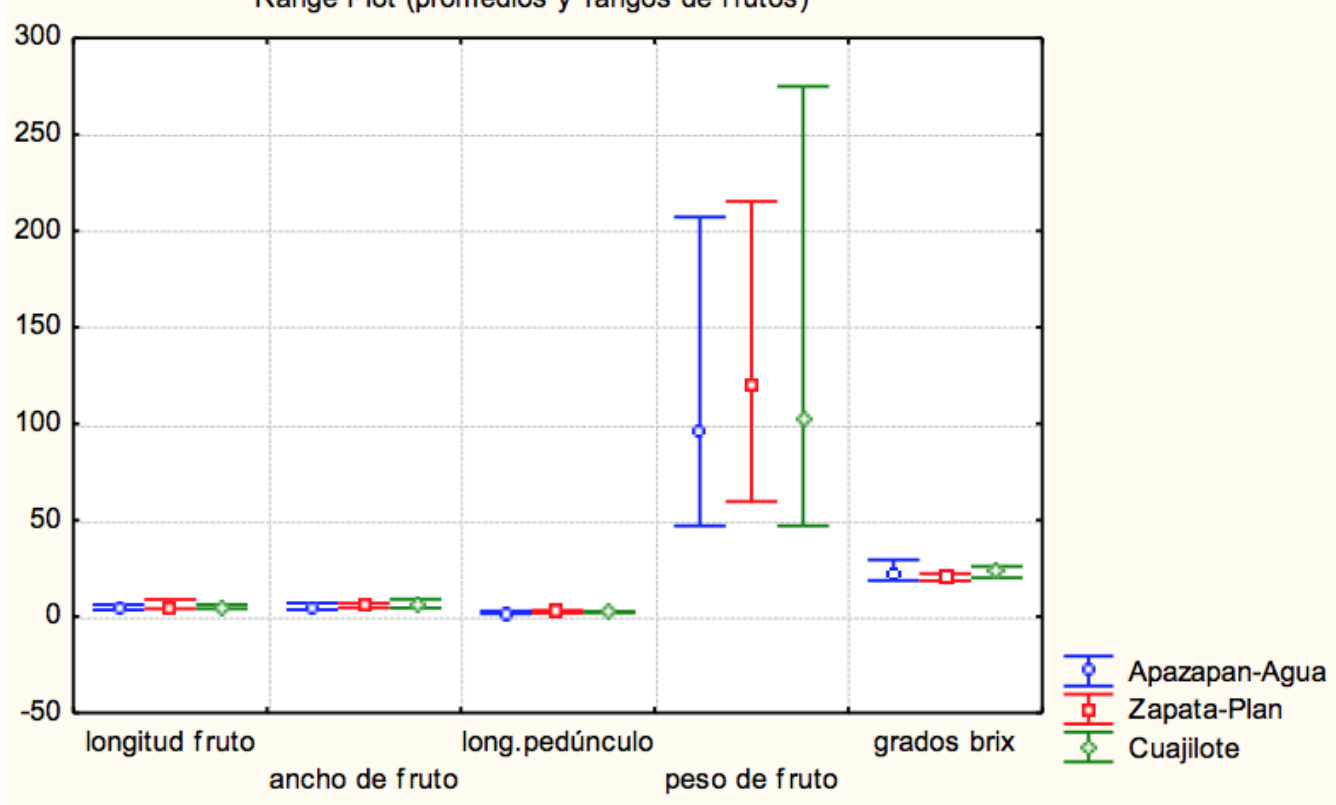

Figura 3. Promedios y rangos por región de colecta, de las variables numéricas de frutos.

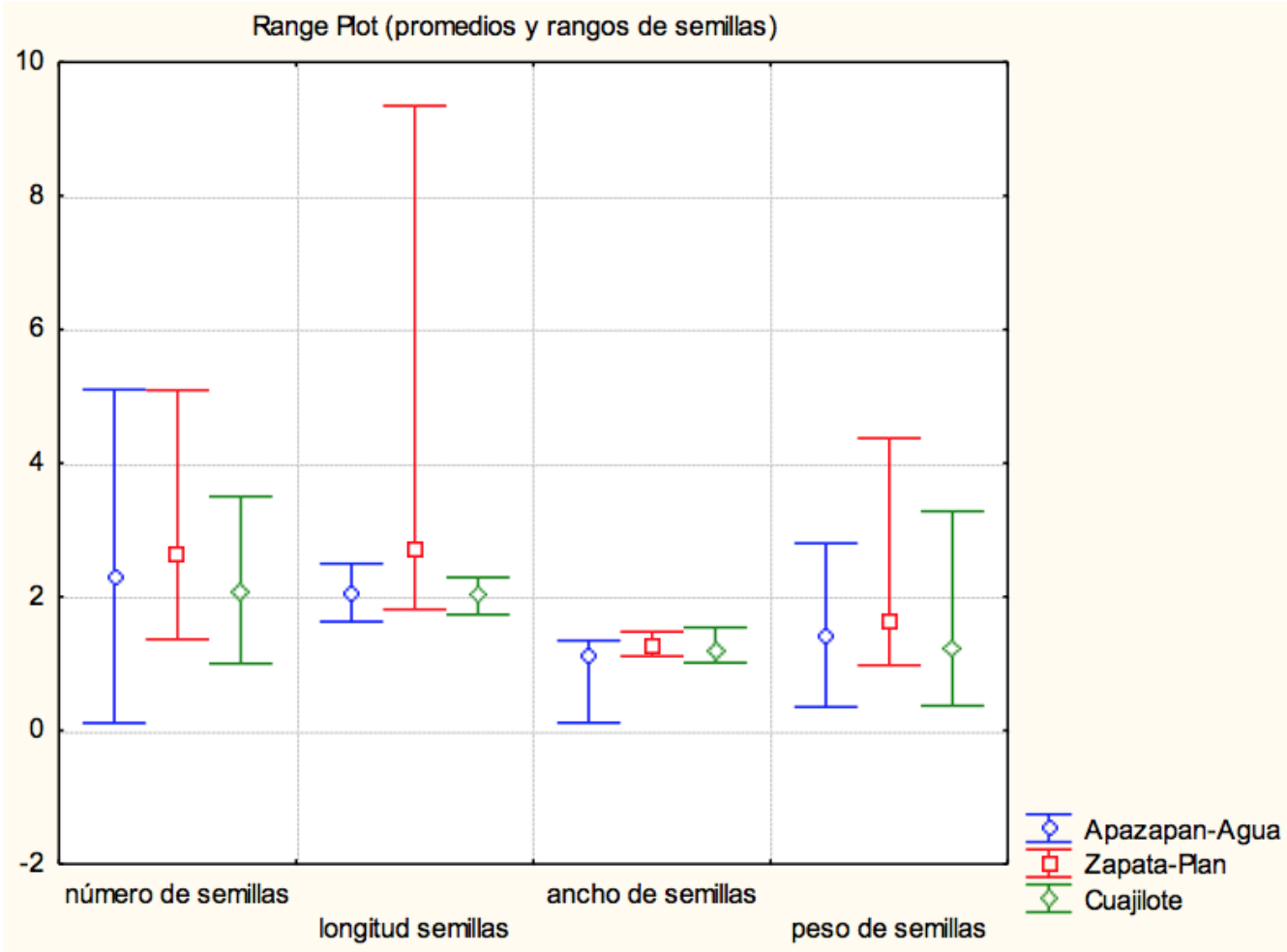

Figura 4. Promedios y rangos por región de colecta, de las variables numéricas de semillas. 
Tabla 3. Matriz de correlación con eigenvalores del análisis de componentes principales, su proporción y la proporción acumulativa de mayor explicación al modelo que caracterizan al chicozapote criollo en el centro de Veracruz

\begin{tabular}{|c|c|c|}
\hline Eigenvalores & Proporción & Proporción Acumulativa \\
\hline 6.15 & 0.34 & 0.3416 \\
2.92 & 0.16 & 0.5040 \\
2.19 & 0.12 & $\mathbf{0 . 6 2 6 0}$ \\
1.66 & 0.09 & 0.7183 \\
1.18 & 0.06 & 0.7836 \\
\hline
\end{tabular}

En la tabla 4 se muestran los componentes principales que explican la mayor variabilidad de los chicozapotes criollos muestreados. Los rasgos botánicos que tienen mayor explicación se ubican en los tres componentes principales o vectores de mayor peso. El primer componente (PRIN1) posee las variables explicadoras de mayor peso, con una proporción de 0.3416; el segundo componente (PRIN2) participa con una porción de 0.1624; el tercer componente (PRIN3) lo hace con una proporción de 0.1219 .

En la figura 5 se observa cómo los árboles colectados en el estado de Morelos (M1 a M5) se ubican en el primer cuadrante positivo y se separan completamente de los árboles colectados en el Estado de Veracruz. Lo que indica que la diferencia geográfica también se asocia al fenotipo expresado por los árboles criollos de chicozapote en ambas regiones de colecta. Los caracteres morfológicos seleccionados pueden variar según el aprovechamiento del recurso natural que representa esta especie en particular (fruto, látex o madera). En el fruto se encontró una gran variabilidad en la forma, color, textura del fruto y de su pulpa, y grados brix en los árboles de chicozapote criollo muestreados.

En el análisis canónico discriminante (ACD), los coeficientes de correlación fueron de 0.70 para el primer y tercer componente principal, y 0.60 para el segundo, a pesar de que sólo incluyen las variables ancho de hoja, ancho de fruto, longitud del pedicelo del fruto y peso del fruto para el primer componente; longitud del pedicelo de la hoja y número de semillas para el segundo componente, así como flores por racimo y diámetro de la flor para el tercer componente. Esto indica la importancia relativa de dichas variables para la caracterización del chicozapote.

En la tabla 5 se observa que con el análisis canónico discriminante se consideran las variables o rasgos botánicos más relevantes, como son ancho de hoja, flores por racimo, ancho 
Tabla 4. Tres componentes principales (PRIN1, PRIN2 y PRIN3) que explican $62.6 \%$ de la variabilidad acumulada en chicozapote criollo de la zona Central del estado de Veracruz y del estado de Morelos, México

\begin{tabular}{|c|c|c|c|}
\hline \multicolumn{3}{|c|}{ Eigenvectores } \\
\hline Variables & PRIN1 & PRIN2 & PRIN3 \\
\hline Hojas por racimo & -.264751 & 0.292121 & 0.114587 \\
Longitud de hoja & 0.200916 & $-\mathbf{0 . 3 2 6 4 6 8}$ & 0.208937 \\
Ancho de hoja & $\mathbf{- 0 . 3 0 5 5 7 7}$ & 0.297764 & 0.191810 \\
Longitud del pedicelo de la hoja & 0.113512 & -0.129382 & $\mathbf{0 . 3 9 8 3 7 4}$ \\
Flores por racimo & 0.022554 & -0.031367 & $\mathbf{0 . 5 5 4 1 2 2}$ \\
Diámetro del botón floral & -0.211223 & 0.122077 & $\mathbf{0 . 3 7 9 6 1 8}$ \\
Diámetro de la flor & -0.009023 & -0.018142 & $\mathbf{0 . 4 9 7 3 1 3}$ \\
Longitud de la flor & 0.262567 & -0.270770 & 0.179677 \\
Longitud del pedicelo de la flor & -0.166238 & $\mathbf{0 . 3 5 7 0 8 5}$ & -0.008703 \\
Longitud del fruto & 0.229925 & 0.209790 & -0.014463 \\
Ancho del fruto & $\mathbf{0 . 3 1 6 9 8 4}$ & 0.230095 & 0.012316 \\
Longitud del pedúnculo del fruto & $\mathbf{0 . 3 5 3 0 2 3}$ & 0.173408 & -0.022066 \\
Peso del fruto & $\mathbf{0 . 3 2 5 7 1 3}$ & 0.222622 & 0.035486 \\
Grados brix & -0.227037 & -0.167527 & -0.104732 \\
Número de semillas & -0.116929 & $\mathbf{0 . 3 8 8 1 3 4}$ & 0.022112 \\
Longitud de semilla & 0.250929 & 0.169255 & -0.026921 \\
Ancho de semilla & 0.269992 & 0.261136 & 0.015595 \\
Peso de semilla & 0.237396 & 0.183027 & 0.048459 \\
\hline
\end{tabular}

de fruto, longitud del pedúnculo y peso del fruto; los cuales proporcionan la mayor explicación y son los que más influyen en la caracterización de los materiales criollos regionales de chicozapote.

En la figura 6 se observa que las muestras de los árboles del Estado de Morelos (M1 a M5) se separan claramente de las muestras de la región central de Veracruz, tanto de los ár- boles criollos de Apazapan (A1 a A16), Agua Caliente (G1, G2 y G3), Emiliano Zapata-Plan del Río (Z1 a Z11) y Cuajilote-San José Chipila (C1 a C8); así como de la variedad Betawi (B1 y B2), a pesar de considerarse los mismos caracteres botánicos. Esto sugiere que los criollos provienen del mismo centro de origen o probablemente la variación se deba al entrecruzamiento natural a través del tiempo. 
Tabla 5. Coeficientes canónicos estandarizados que identifican las variables más importantes que dan la mejor explicaron de la varianza observada

\begin{tabular}{|c|c|c|c|}
\hline \multicolumn{4}{|c|}{ Eigenvectores } \\
\hline Variables & $\begin{array}{c}\text { Coeficiente } \\
\text { canónico 1 }\end{array}$ & $\begin{array}{c}\text { Coeficiente } \\
\text { canónico 2 }\end{array}$ & $\begin{array}{c}\text { Coeficiente } \\
\text { canónico 3 }\end{array}$ \\
\hline Ancho de hoja & $\mathbf{2 . 9 0 7 8 0 0}$ & 0.241891 & -0.900244 \\
Flores por racimo & -0.126181 & $\mathbf{1 . 3 3 3 5 7 5}$ & 0.936127 \\
Diámetro de flor & -0.033897 & -0.580874 & -0.245845 \\
Longitud de pedicelo flor & 0.493524 & 0.253006 & 0.660882 \\
Ancho de fruto & -0.373874 & $\mathbf{1 . 7 3 4 6 7 0}$ & $\mathbf{- 1 . 7 5 3 9 8 5}$ \\
Longitud pedúnculo fruto & -0.273658 & $\mathbf{1 . 0 5 0 9 4 5}$ & $\mathbf{1 . 2 4 4 5 5 9}$ \\
Peso del fruto & 0.603787 & $\mathbf{- 2 . 2 0 6 9 0 3}$ & 0.014493 \\
Número de semillas & -0.035883 & -0.365855 & 0.652046 \\
\hline
\end{tabular}

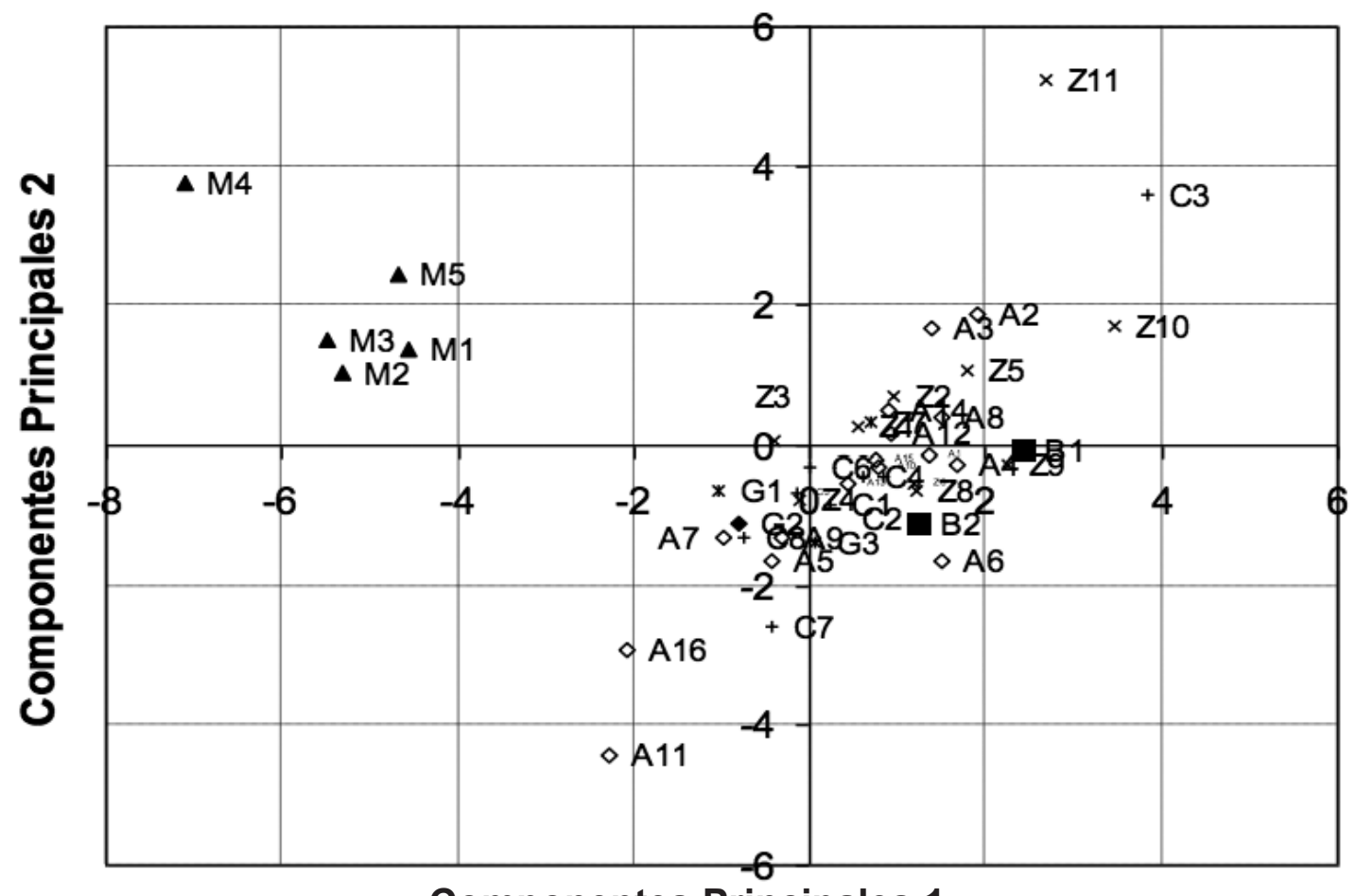

Componentes Principales 1

Figura 5. Representación de la variabilidad de los caracteres morfológicos de árboles muestreados en la región centro del Estado de Veracruz y del Estado de Morelos, México. X = componente principal 1 (PRIN1), Y = PRIN2. Ver las claves de cada árbol en la tabla 2. 
Tabla 6. Valores acumulados de la correlación canónica que dan una mayor explicación al modelo de caracterización del chicozapote criollo en Veracruz y Morelos, México

\begin{tabular}{|c|c|c|}
\hline Eigenvalor & Proporción & Acumulativo \\
\hline 10.7330 & 0.8470 & 0.8470 \\
1.0270 & 0.0811 & 0.9281 \\
0.5062 & 0.0399 & 0.9680 \\
0.3227 & 0.0255 & 0.9935 \\
0.0822 & 0.0065 & 1.0000 \\
\hline
\end{tabular}

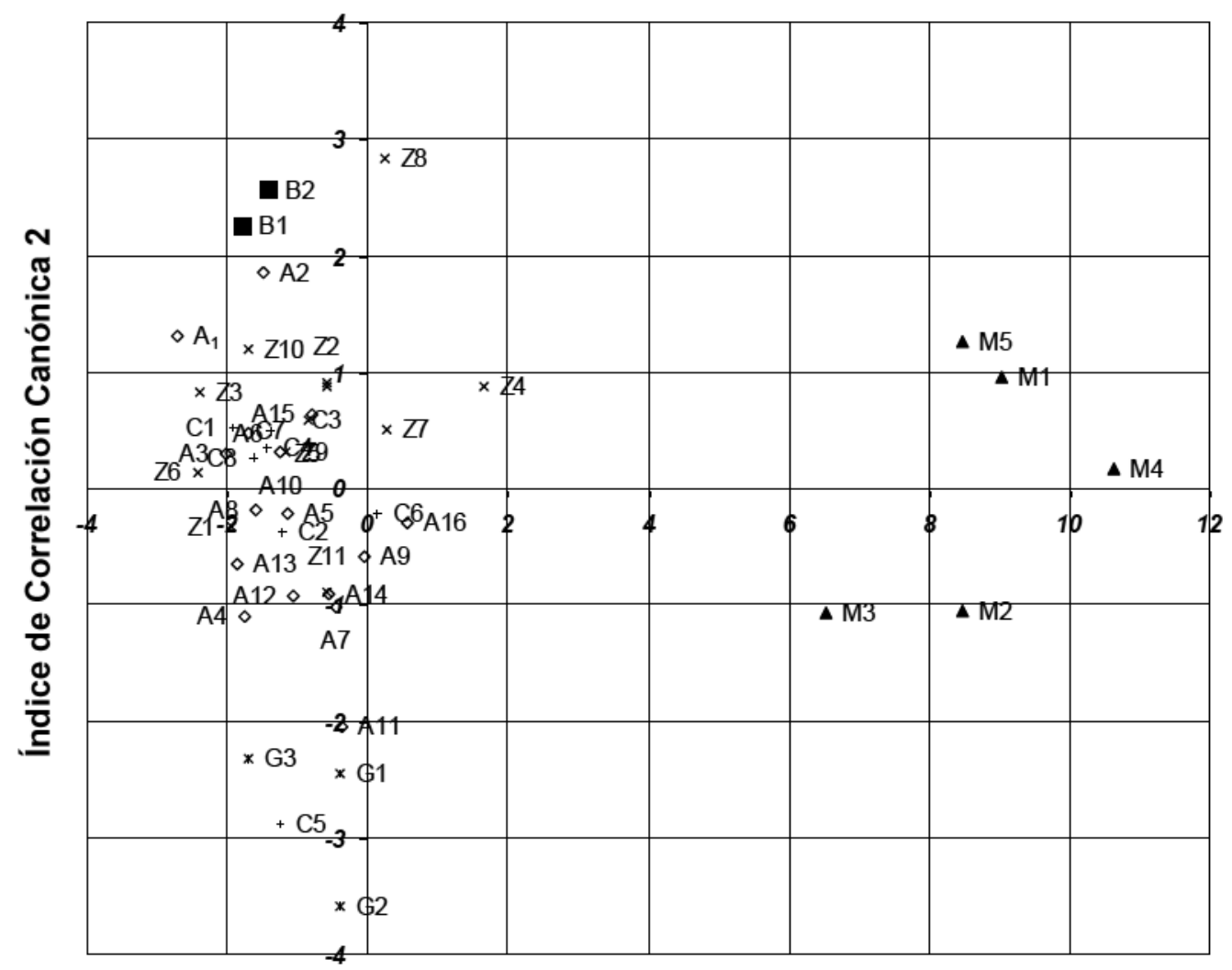

Índice de Correlación Canónica 1

Figura 6. Ubicación cartesiana de los árboles de chicozapote por localidad con relación a los índices de correlación canónica 1 (eje X) y 2 (eje Y). 
En el análisis de conglomerados se obtuvo un dendograma (figura 7) sobre la base de 43 árboles de chicozapote criollo y dos de chicozapote mejorado muestreados, resultando varios grupos. Los árboles de chicozapote formaron cuatro grupos principales. Los criollos del Estado de Morelos presentan baja semejanza con el resto de los grupos de la región centro del Estado de Veracruz, dicho patrón morfológico marca un posible origen diferente.

En general, los criollos del centro de Veracruz presentaron valores del coeficiente de diferenciación bajos, y forman parte del mismo ramal principal. Tres grupos muestran un coeficiente bajo (0.01 a 0.02), lo que permite inferir que hay similitud entre los criollos de Apazapan, E. Zapata, Cuajilote y Agua Caliente. En el grupo donde se encuentra la variedad mejorada introducida, se observan materiales criollos interrelacionados con dicha variedad provenientes de Apazapan y E. Zapata, lo que indica que su origen sea posiblemente de un criollo con características similares a los del centro de Veracruz.

En un análisis molecular con micro satélites de 20 árboles del Estado de Veracruz; González-Hernández et al. (2012) también determinaron el agrupamiento de ejemplares del centro y sur del Estado, lo cual pudo explicarse por el movimiento de la especie por agricultores en busca de nuevas áreas de producción; o por los pobladores que la han trasladado para su consumo en la región.
Afortunadamente, se han conformado redes a nivel nacional para colectar y conservar la variabilidad de M. zapota (Villegas et al., 2013), lo cual permitirá retomar este tipo de materiales para su eventual selección, mejoramiento y reproducción; permitiendo explotar nichos comerciales especiales con características relevantes, en especial con los frutos de muy poca semilla, tamaño pequeño tipo gourmet y altos grados brix.

En futuras colectas del frutal, es conveniente considerar los caracteres morfológicos que en la matriz de correlación de componentes principales dieron la mayor explicación (Ver figura 7).

\section{Conclusiones}

Los rasgos botánicos morfológicos ancho de hoja, flores por racimo, ancho de fruto, longitud del pedúnculo del fruto y peso del fruto caracterizan los materiales criollos de chicozapote. El grupo de árboles del Estado de Morelos difiere del presente en la zona centro de Veracruz, mientras que los árboles de la variedad Betawi coinciden más con uno de los grupos de esta zona.

Las características de los criollos del centro de Veracruz son: racimos, longitud de ancho y de pedicelo de hoja de $12.5,10,4.6$ y $2.3 \mathrm{~cm}$, respectivamente; de forma lanceolada, color verde oscura, textura lisa y posición alterna de la hoja. Flores en racimo, diámetro de 


\section{Diagrama de árbol para 45 variables} Ligamiento completo

Distancias Euclideanas

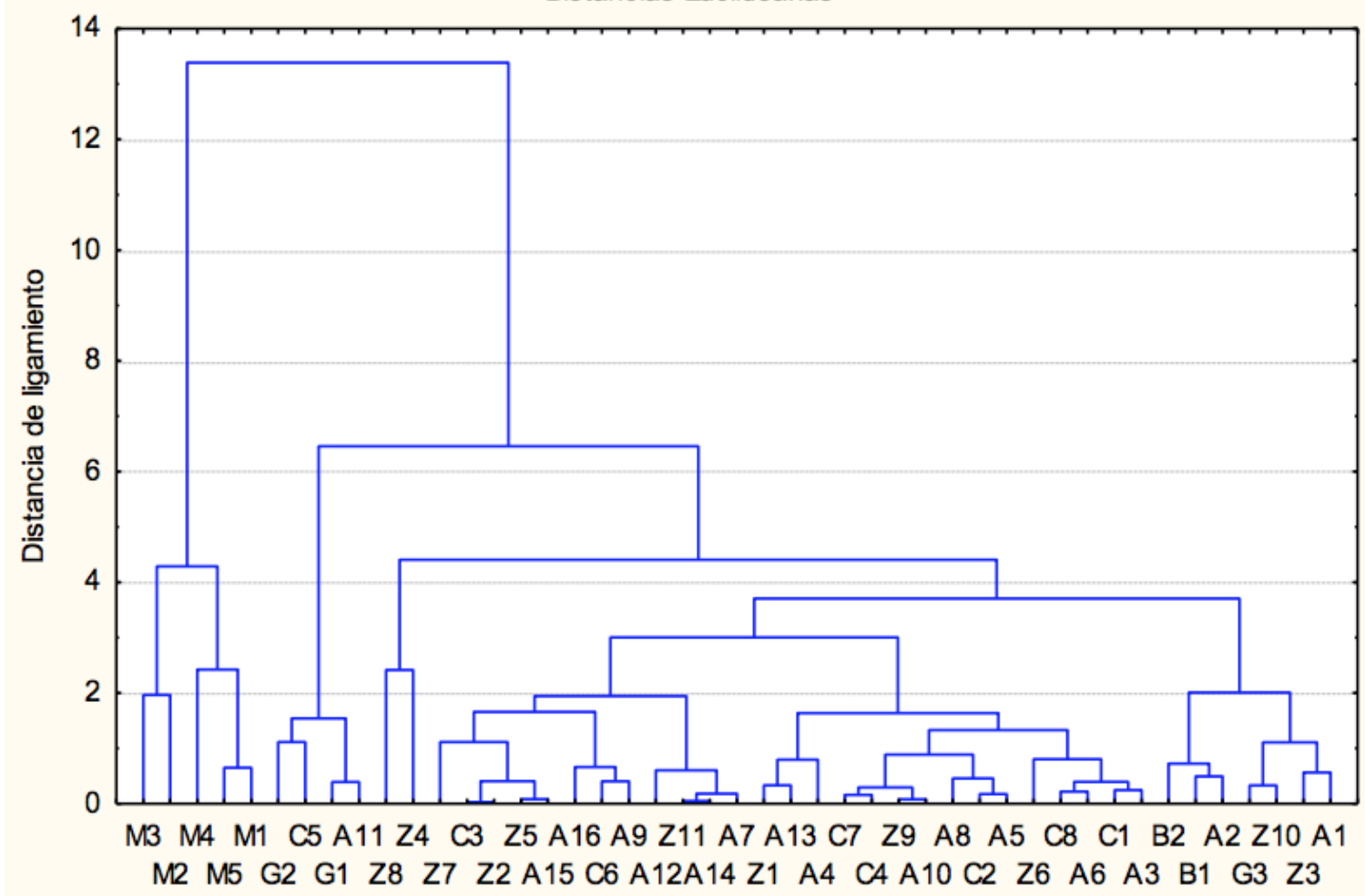

Figura 7. Dendograma por clúster para el chicozapote criollo de la región centro del Estado de Veracruz, del Estado de Morelos, y de la variedad mejorada Betawi.

botón y flor, longitud de flor y pedicelo con medidas de $5.9,0.55,0.69,1$ y $1.72 \mathrm{~cm}$, respectivamente, y un color crema de pétalo. En cuanto a fruto, 22.21 grados brix, así como $5,5.23$ y $2.21 \mathrm{~cm}$ de longitud, ancho y longitud de pedúnculo, respectivamente; así como $100 \mathrm{~g}$ de peso; forma redonda, color café oscuro, textura rugosa y de pulpa café claro. En las 2.5 semillas por fruto, las medidas son 2.16 y $1.14 \mathrm{~cm}$ de longitud y anchura, y $1.29 \mathrm{~g}$ de peso, con las variables categóricas color café y oscuro, y textura lisa.
Existen árboles criollos de características relevantes que le confieren una calidad excepcional al fruto, tales como forma ovalada o semiovalada; color café claro, poco rugoso, con pulpa café rojiza sin grumos; tamaño mediano a grande y un número escaso de semillas. Estos materiales deben rescatarse para formar a futuro una variedad mejorada. 


\section{Referencias}

Báez L, A. (1979). Generalidades del chicozapote. CONAFRUT, CEEIF. Xalapa, Veracruz, México

Batis M, A.I; Alcocer S, M.I; Gual D, M; Sánchez D, C y Vázquez Y, C. (1999). Árboles mexicanos potencialmente valiosos para la restauración ecológica y la reforestación. Universidad Nacional Autónoma de México-Instituto de Ecología. Informe final SNIB-CONABIO proyecto No. J084. México, D. F. p. 239-242. Recuperado de http://www.conabio.gob.mx/ conocimiento/info_especies/arboles/doctos/64-sapot4m.pdf

Castner, J.L; Timme, S.L y Duke, J.A. (1998). A field guide to medicinal and useful plants of the upper Amazon. Feline Press, Gainesville, Florida, USA

García, C.X; Rodríguez, S.B y Parraguirre L, C. (1993). Notas importantes sobre el chicozapote (Manilkara zapota (L.) Van Royen). Ciencia For. 18 (74): 1-166.

González-Hernández, D; García-Pérez, E; Guntin-Marey, P. (2012). Caracterización genética de Manilkara zapota de Veracruz, México, con marcadores SSR. Agrociencia, 46(7): 663-675

Hernández B, J.E. y León, J. (eds.) (1992). Cultivos marginales, otra perspectiva de 1492. Colección FAO: Producción y protección vegetal $\mathrm{N}^{\circ} 26$. Organización de las Naciones Unidas para la Agricultura y la Alimentación. Roma, Italia

Herron, S. (1999). The Economic Botanic of Manilkara zapota (L.) Van Royen. Etnobotanical Leaflets. Southern Illinois University Carbondale, USA

León, J. (1968). Fundamentos botánicos de los cultivos tropicales. Instituto Interamericano de Ciencias Agrícolas de la Organización de los Estados Americanos, San José de Costa Rica. Cap. 19. p. 244-246

Ojeda R, M.M. (2000). Una introducción a los métodos multivariados. Facultad de Estadística e Informática. Especialización en Métodos Estadísticos. Universidad Veracruzana. Xalapa, México

Ortega, P.R; Martínez A., M.A. y Sánchez G, J.J. (2000). Recursos fitogenéticos autóctonos. En: Recursos Fitogenéticos de México para la Alimentación y la Agricultura. SAGAR, SNICS, Sociedad Mexicana de Fitogenética. Chapingo, México. p. 43. 
Ramírez V, P; Ortega P, R; López H, A; Castillo G, F; Livera M, M; Rincón S, F. y Zavala G, F. (eds). (2000). Recursos fitogenéticos de México para la alimentación y la agricultura, Informe Nacional. Servicio Nacional de Inspección y Certificación de Semillas-Sociedad Mexicana de Fitogenética A.C. Chapingo, México. p. 188.

Roecklein, J.C. y Leung, P.S. (1987). A profile of economic plants. Transaction, Inc., New Brunswick, New Jersey, USA. p. 629.

Rzedowski, J. (1988). Diversidad y origen de la flora fanerógama de México. En: Ramamoorty, TP; Bye, R; Lot, A; Fa, J. (comp.). Diversidad Biológica en México, Origen y Distribución. UNAM, Instituto de Biología, México. p. 129-145.

Toledo, V.M. (1982). Pleistocene changes of vegetation in tropical Mexico. pp. 93-112. In: G. T. Prance, editor. Biological Diversification in the Tropics. Columbia Univ. Press, New York, USA.

Villegas M, A; Cituk C, D; Arrieta R, G; Espinosa Z, S. y Damián N, A. (2013). Selección, caracterización y uso de sapotáceas. Programa Nacional de Selección, Caracterización, Conservación y Uso de los Recursos Genéticos de Sapotáceas (Pouteria sapota, Manilkara zapota y Chrysopyllum cainito). In: Servicio Nacional de Inspección y Certificación de Semillas. Resúmenes Ejecutivos Ejercicio Fiscal 2010. Sistema Nacional de Recursos Fitogenéticos para la Alimentación y la Agricultura. Editorial Universidad Autónoma Chapingo. Texcoco, Edo. de México. p. 145-147. 